\title{
Multifaceted Beneficial Effects of Erdosteine: More than a Mucolytic Agent
}

\author{
Mario Cazzola ${ }^{1}$ (D) ${\text { Clive } \text { Page }^{2} \cdot \text { Paola Rogliani }}^{1} \cdot$ Luigino Calzetta $^{3} \cdot$ Maria Gabriella Matera $^{4}$
}

Published online: 6 October 2020

(c) The Author(s) 2020

\begin{abstract}
Erdosteine is a drug approved for the treatment of acute and chronic pulmonary diseases, originally developed as a mucolytic agent. It belongs to the thiol-based family of drugs that are known to also possess potentially important antioxidant and anti-inflammatory properties, and exhibit antibacterial activity against a variety of medically important bacterial species. Erdosteine is a prodrug that is metabolized to the ring-opening compound metabolite M1 (MET 1), which has mucolytic properties. Experimental studies have documented that erdosteine prevents or reduces lung tissue damage induced by oxidative stress and, in particular, that Met 1 also regulates reactive oxygen species production. The RESTORE study, which has been the only trial that investigated the effects of a thiol-based drug in chronic obstructive pulmonary disease (COPD) frequent exacerbators, documented that erdosteine significantly reduces the risk of acute exacerbations of COPD (AECOPDs), shortens their course, and also decreases the risk of hospitalization from COPD. The preventive action of erdosteine on AECOPDs was not affected by the presence or absence of inhaled corticosteroids (ICSs) or blood eosinophil count. These findings clearly contrast with the Global Initiative for Chronic Obstructive Lung Disease strategy's approach to use erdosteine only in those COPD patients not treated simultaneously with an ICS. Furthermore, they support the possibility of using erdosteine in a step-down approach that in COPD is characterized by the withdrawal of the ICS.
\end{abstract}

\section{Introduction}

Erdosteine [ $N$-(carboxymethylthioacetyl)-homocysteine thiolactone] is a thiol-based drug classified as a mucolytic agent [1]. Thiols are the sulfur analogues of alcohols characterized by the presence of sulfhydryl (-SH) groups at their active site. They stabilize the tertiary and quaternary structures of proteins by forming intra- and inter-chain disulfide bonds (S-S), play many roles in metabolism and homeostasis, and are important in several physiological and pathological processes [2]. Erdosteine contains two sulfur atoms, one of

Mario Cazzola

mario.cazzola@uniroma2.it

1 Unit of Respiratory Medicine, Department of Experimental Medicine, University of Rome "Tor Vergata", Rome, Italy

2 Sackler Institute of Pulmonary Pharmacology, Institute of Pharmaceutical Science, King's College London, London, UK

3 Unit of Respiratory Disease and Lung Function, Department Medicine and Surgery, University of Parma, Parma, Italy

4 Unit of Pharmacology, Department of Experimental Medicine, University of Campania "Luigi Vanvitelli", Naples, Italy which is a thioether in the aliphatic side chain and the other is enclosed in the heterocyclic ring (thiolactone). These two -SH groups are blocked, but erdosteine is metabolized in the liver to the ring-opening compound, metabolite M1 (Met 1) or ( \pm )- $N$-(2-carboxymethylthioacetyl)homocysteine, which contains a free pharmacologically active $-\mathrm{SH}$ group in its chemical structure [3] (Fig. 1).

Thiol-based drugs, in particular erdosteine, $\mathrm{N}$-acetyl L-cysteine (NAC) and $S$-carboxymethylcysteine, clearly exhibit multiple pharmacological actions of relevance to the treatment of a range of respiratory diseases including chronic obstructive pulmonary disease (COPD), asthma, and possibly idiopathic pulmonary fibrosis (IPF) [2]. They have traditionally been considered as mucolytic drugs because they decrease the viscosity, and mostly the elasticity, of bronchial secretions by reducing $\mathrm{S}-\mathrm{S}$ bonds in proteins in mucous. However, thiol-based drugs can also act as antioxidant drugs directly through free - $\mathrm{SH}$ groups that serve as a source of reducing equivalents, as well as indirectly through the replenishment of intracellular glutathione (GSH) levels. Moreover, thiol-based drugs interfere with inflammatory pathways and can modulate the tone of airway smooth muscle in human bronchi. There is also evidence that thiolbased drugs can reduce bacterial adhesion to the respiratory 


\section{Key Points}

Erdosteine is a thiol-based drug classified as a mucolytic agent. However, it can also act as an antioxidant drug, interferes with inflammatory pathways, modulates human bronchial tone, and has anti-infective properties.

A landmark study investigating the effects of a thiolbased drug in COPD frequent exacerbators documented that erdosteine significantly reduces the risk of AECOPDs, shortens their course, and also decreases the risk of hospitalization from COPD.

The preventive effect on the risk of AECOPD exerted by erdosteine is present both in patients who are being treated with ICSs and in those who do not use this treatment, and, in any case, it is not affected by the blood count of eosinophils.

It has been calculated that 10.11 patients have to be treated with erdosteine for 1 year to prevent one AECOPD, compared to placebo, while the NNT values for both $S$-carboxymethylcysteine (30.92) and NAC (15.69) are not significantly different to placebo.

epithelial cell surface and inhibit biofilm formation, causing biofilm disruption and thereby improving the efficacy of antibiotic therapy [2]. The documented activities of these thiol-based drugs are summarized in Table 1.

Erdosteine was first comprehensively reviewed in 1996 [1]. In this article, we review the considerable amount of new information on erdosteine that has emerged since the 1996 publication. We searched EMBASE, MEDLINE, Scopus, The Cochrane Library, Web of Science, and Google Scholar from January 1997 through May 2020. We supplemented the bibliographic database searches with backward citation tracking of relevant publications. We also searched https://www.clinicaltrials.gov for on-going studies and selected the most significant information.

\section{Update on the Pharmacology of Erdosteine}

The well-established mucolytic activity of erdosteine has been supported by further studies that have investigated its influence on mucin solution in vitro, on mucociliary transport in quails, on airway secretions in rats and on cough reflexes in guinea pigs [4]. In quails, erdosteine, $600 \mathrm{mg} /$ $\mathrm{kg}$, but not $300 \mathrm{mg} / \mathrm{kg}$, significantly promoted mucociliary transport, while changes in the mucociliary transport rates of the group treated with $S$-carboxymethylcysteine were similar (a)<smiles>O=C(O)CSCC(=O)NC1CCSC1=O</smiles>

(b)

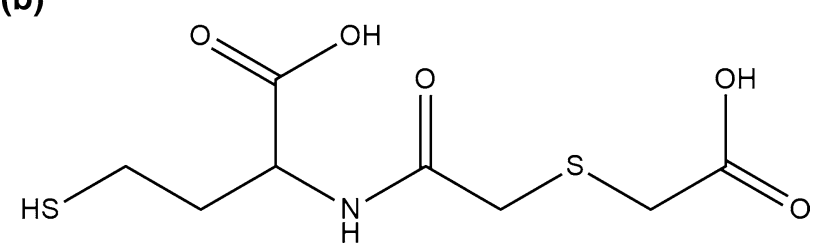

Fig. 1 Chemical structure of erdosteine (a) and Met I (b)

to those of the control group. Furthermore, erdosteine significantly increased airway secretions in rats, an effect that was still apparent $24 \mathrm{~h}$ after its administration. Erdosteine also significantly suppressed citric acid-induced cough reflexes in guinea pigs, but importantly did not suppress mechanical stimuli-induced cough reflexes. This finding is intriguing because it suggests that erdosteine only reduces cough induced by irritants, as would be expected to be associated with airway inflammation, without suppressing the homeostatic cough reflex triggered by mechanical stimuli such as the presence of mucous plugs, thus allowing sputum expulsion. It is of interest that mucociliary clearance can be inhibited in airway inflammatory disease [5], but in another study assessing the effect of erdosteine on the mucociliary clearance in normal and airway-inflammation-induced rats, this drug was able to promote mucociliary clearance in both circumstances [6].

Since the mucolytic activity of erdosteine has been well documented, more recently attention has been focused on the antioxidant and anti-inflammatory properties of this molecule.

Erdosteine exerts an antioxidant effect on neutrophils and eosinophils because of the scavenging effect of its metabolite, Met 1. Scavenging activities of erdosteine and Met 1 against $\mathrm{O}_{2}{ }^{-}$anion, hydrogen peroxide $\left(\mathrm{H}_{2} \mathrm{O}_{2}\right)$ and hypochlorous acid $(\mathrm{HOCl})$ were evaluated. Erdosteine, as a prodrug, did not reduce reactive oxygen species (ROS), but Met 1 substantially scavenged $\mathrm{H}_{2} \mathrm{O}_{2}$ and $\mathrm{HOCl}$ in vitro [7].

The rate constants of tested drugs were estimated by comparison with that of GSH. The affinity of Met 1 was $80 \%$ of that of GSH, while NAC was 70\% [7]. Met 1 has also been demonstrated to regulate ROS produced by rat neutrophils, guinea-pig eosinophils and human neutrophils [3]. The effects of $S$-carboxymethylcysteine, ambroxol and NAC were weaker than those of Met 1 . The antioxidant effect of 
Table 1 The documented activities of the main thiol-based drugs (modified from Cazzola et al. [2])

\begin{tabular}{|c|c|c|c|}
\hline & Erdosteine & NAC & $\begin{array}{l}S \text {-Carbox- } \\
\text { ymethyl- } \\
\text { cysteine }\end{array}$ \\
\hline \multicolumn{4}{|l|}{ Mucolytic activity } \\
\hline Reduced viscosity of mucoprotein solutions & + & + & + \\
\hline Reduced bronchial secretions & & + & + \\
\hline Increased mucociliary clearance & + & + & + \\
\hline \multicolumn{4}{|l|}{ Antioxidant activity } \\
\hline Reduced pro-oxidant profile & + & + & + \\
\hline Increased antioxidant profile & + & + & + \\
\hline \multicolumn{4}{|l|}{ Anti-inflammatory activity } \\
\hline Reduced neurogenic inflammation & & + & \\
\hline Reduced cytokine release & + & + & + \\
\hline Reduced proteinase synthesis & + & + & \\
\hline $\begin{array}{l}\text { Reduced levels of proinflammatory proteins and activation of } \\
\text { transcription factors }\end{array}$ & + & + & + \\
\hline \multicolumn{4}{|l|}{ Direct and indirect antibacterial activity } \\
\hline Increased neutrophil killing activity & & + & \\
\hline Bacteriostatic effect & & + & \\
\hline Reduced bacterial adhesion on epithelium & + & + & + \\
\hline Reduced biofilm formation & & + & \\
\hline Improved antibiotic activity & + & \pm & + \\
\hline Viral replication and infectivity & & + & + \\
\hline
\end{tabular}

+ Demonstrated activity; \pm inconsistent activity; empty cells indicate that the activity has not been investigated

Met 1 was confirmed in a study that showed a significant concentration-dependent inhibition of oxidative bursts in human neutrophils comparable to the inhibition observed with the control drug NAC [8]. Met 1 was also effective in preventing $\mathrm{H}_{2} \mathrm{O}_{2}$-induced oxidative stress and DNA damage in A549 human lung adenocarcinoma cells by the scavenging of intracellular ROS [9]. It has been documented that the $-\mathrm{SH}$ group of Met 1 is capable of reducing the amount of $\mathrm{N}$-centred radical species because of a termination reaction between the free radicals and Met 1 as a mechanism to explain the antioxidant effect of this drug [10]. Erdosteine has also been demonstrated to prevent or reduce lung tissue damage induced by oxidative stress in a variety of experimental models [11-13].

Since there is evidence that ROS generation initiates the activation of various signalling pathways and ultimately induces the expression of inflammatory proteins [14] (Fig. 2), it is really not surprising that erdosteine also elicits anti-inflammatory effects.

Thus, erdosteine has been shown to inhibit lipopolysaccharide (LPS)-induced nuclear factor- $\mathrm{\kappa B}$ (NF- $\mathrm{\kappa B}$ ) activation in mouse macrophages [15] and tumor necrosis factor- $\alpha$ (TNF- $\alpha$ ), interleukin (IL)-1 $\beta$ and free radical production in rat alveolar macrophages [16]. Hayashi et al. reported that erdosteine inhibited the LPS-induced neutrophil influx in a mouse lung injury model [17] and it has also been shown that it inhibited LPS-induced apoptosis of rat bronchial epithelium [18]. Met 1 can also inhibit the release of elastase from human neutrophils induced by the synthetic chemotactic peptide $N$-formyl-methionyl-leucyl-phenylalanine at concentrations achievable in the clinical setting [19].

Furthermore, erdosteine and Met 1 also exert antibacterial effects by affecting the integrity of the pilin molecules. The interaction between the -SH groups of Met I and the intrachain disulphide bonds of pilins can induce a morphological change in the pilin structure that interferes with the binding of bacterial fimbriae to the receptor.

Met 1 significantly reduced both Staphylococcus aureus and Escherichia coli adhesiveness to human mucosal epithelial cells via inhibition at the fimbrial level [20] (Fig. 3).

The addition of Met 1 to clarithromycin potentiated the inhibition of $S$. aureus adhesiveness to human mucosal cells in comparison with the antibiotic alone [21] (Fig. 4), while the combination of Met 1 and ciprofloxacin potentiated the inhibition of $S$. aureus and E. coli adhesiveness to human mucosal cells in comparison with ciprofloxacin alone [22]. It is likely that the mucolytic activity of erdosteine allows better penetration of antibiotics into the sites of infection, thus positively influencing their antimicrobial effects [2]. 


\section{Clinical Development}

A previous review of the pharmacological actions of erdosteine published in 1996 was based on the few clinical studies available at the time and concluded that orally administered erdosteine is an effective and well tolerated treatment in patients with chronic obstructive bronchial disease, with an efficacy at least equivalent to that of ambroxol in the reduction of symptoms [1].

A consensus panel report of the American College of Chest Physicians (ACCP) on management of cough published in 1998 cited erdosteine as a drug that had been adequately evaluated as a treatment for cough clearance in patients with bronchitis in well-designed clinical studies [23]. Subsequently, in 2006 erdosteine was recommended on a short-term basis to increase cough clearance in patients with bronchitis by the ACCP evidence-based clinical practice guidelines on cough suppressant and pharmacologic protussive therapy [24].

However, already in 1996 it was also recognised that erdosteine clearly exhibits multiple pharmacological actions of relevance for the treatment of stable COPD and has the potential to reduce the risk of acute exacerbation of COPD (AECOPD) [1]. Therefore, since that time there have been considerable efforts to understand the role of erdosteine in patients with stable COPD and in the prevention of exacerbations.

In Table 2 we report the main trials with erdosteine, but have excluded small trials (e.g. less than 60 patients) since those studies do not have statistical power for outcome data, and also anecdotal trials.
Fioretti and Bandera performed a double-blind, randomized, placebo-controlled study in which the effect of 6-month treatment with erdosteine (300 mg twice daily) or placebo was evaluated in 132 patients with chronic bronchitis [25]. Erdosteine significantly reduced the incidence and severity of exacerbations and diminished the number of days absent from work due to exacerbation compared with placebo.

In 237 patients with an infective exacerbation of chronic bronchitis, erdosteine (300 mg twice daily) or placebo in association with amoxicillin $(1500 \mathrm{mg} /$ day $)$ were administered for a maximum of 10 days [26]. Eleven patients

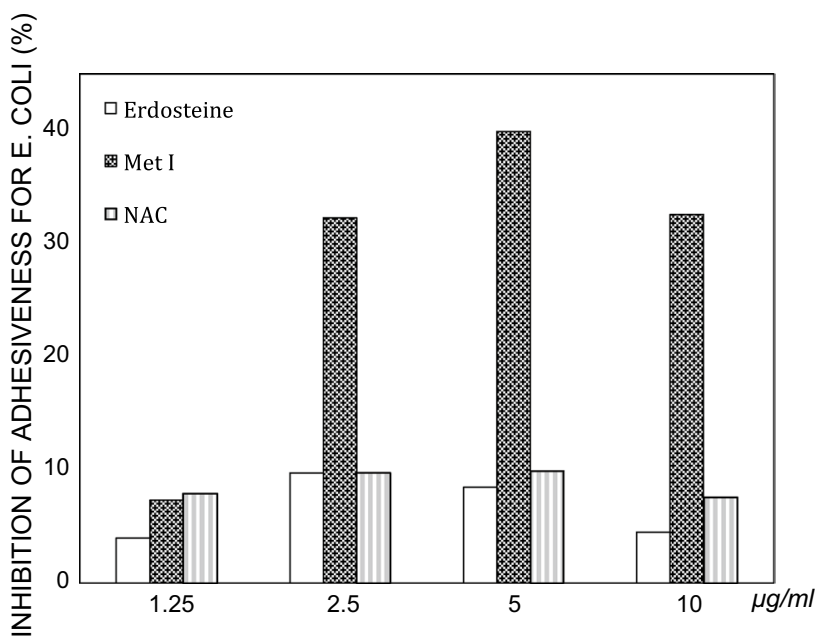

Fig. 3 Comparative analysis of the effect of the drugs on E. coli adhesiveness. The findings are expressed as percentages of the final mean values versus controls (modified from Braga et al. [20])
Fig. 2 Principal signalling pathways caused by reactive oxyden species (ROS) production

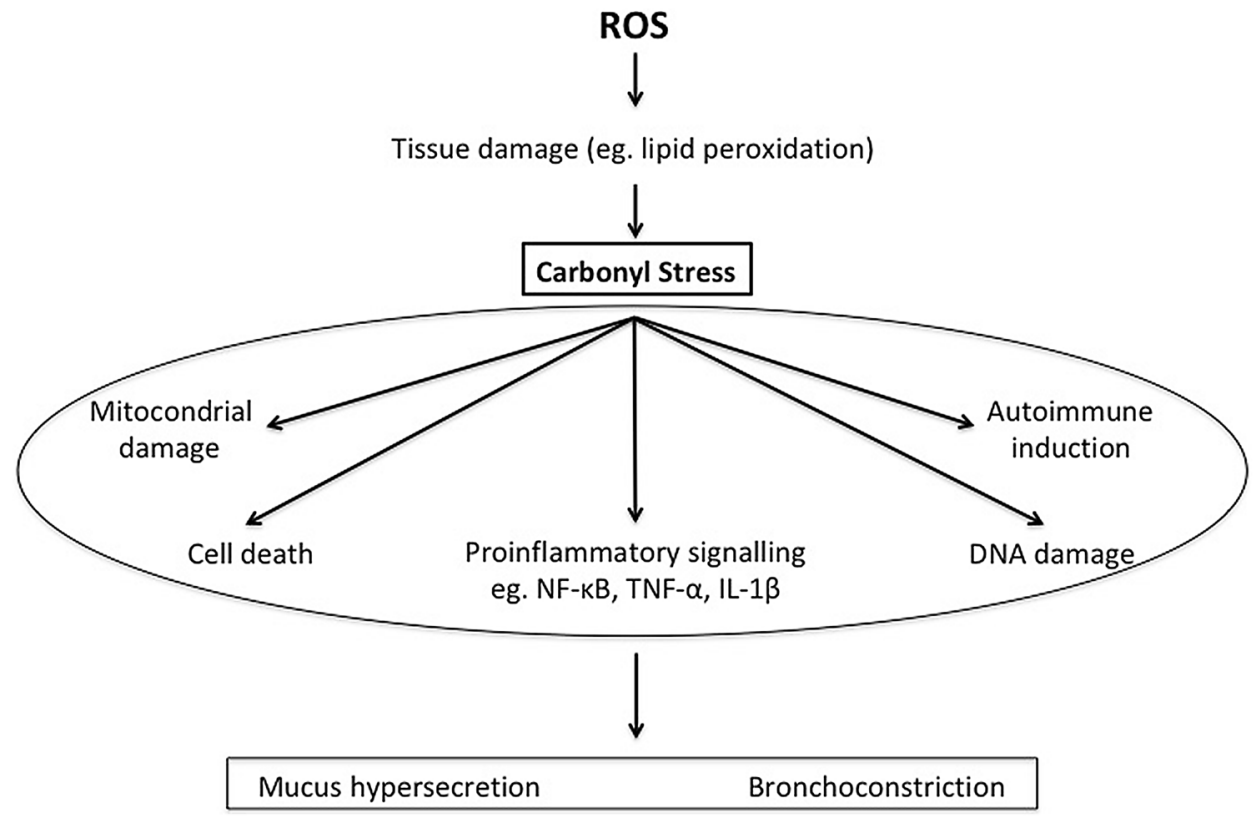


(a)

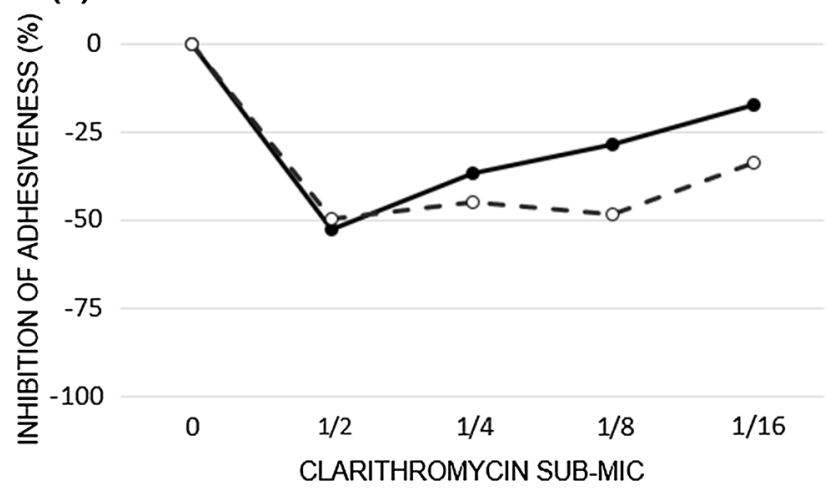

(b)

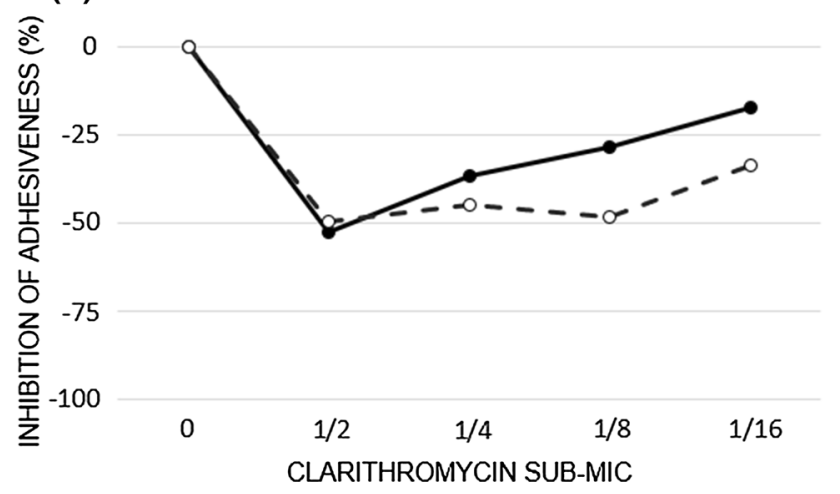

Fig. 4 Effects of sub-MICs of clarithromycin alone (solid lines) and in combination with Met I (dashed lines) $5 \mu \mathrm{g} / \mathrm{mL}$ (a) and $10 \mu \mathrm{g} / \mathrm{mL}$ (b) on S. aureus adhesiveness (modified from Braga et al. [21])

were lost due to different reasons. Clinical symptoms such as breathlessness, cough, and sputum viscosity improved more quickly in patients treated with the combination of amoxicillin and erdosteine compared with those receiving antibiotics alone.

The clinical efficacy of 21 days of treatment with erdosteine $300 \mathrm{mg}$ twice daily was evaluated in 170 stable chronic bronchitis patients with chronic mucus hypersecretion [27]. The global index of efficacy (frequency of the cough + severity of the cough + difficulty in breathing + dyspnoea) and also the intensity and frequency of the cough were statistically significant in favour of erdosteine compared to placebo.

The EQUALIFE study assessed the effectiveness of 8 months of oral treatment with erdosteine $300 \mathrm{mg}$ twice daily in 155 patients with moderate COPD during the winter season [28]. Compared with placebo, erdosteine significantly reduced the number of AECOPDs and hospitalization days, and improved health-related quality of life, with a lower mean total COPD-related disease cost per patient.

It was also documented that a regimen of oral erdosteine $225 \mathrm{mg}$ twice daily in addition to routine chest physiotherapy provided some physiologic and clinical benefits versus physiotherapy alone over a 15-day period in 30 elderly COPD patients with bronchiectasis and chronic mucus hypersecretion, with a significant effect on mucus density, purulence and volume production. The spirometric improvement at the end of the treatment period compared to baseline values was significant in the erdosteine group $(200 \mathrm{~mL}$ in forced expiratory volume in $1 \mathrm{~s}\left(\mathrm{FEV}_{1}\right)$ and $300 \mathrm{~mL}$ in forced vital capacity (FVC)) [29].

These interesting clinical effects of erdosteine in patients with COPD motivated the search for the pharmacological actions of this drug that could support the observed benefits.

An interesting study assessed the effects of erdosteine on smoking-induced lipid peroxidation in healthy smokers and reported that 1 month of treatment significantly decreased the concentration of thiobarbituric acid-reactive substances after smoke exposure, suggesting that this drug might help prevent smoking-induced lipid peroxidation [30].

Erdosteine $(600 \mathrm{mg} / \mathrm{day})$ administered for 10 days in patients with stable mild-to-moderate COPD caused a substantial decline in ROS blood levels and IL-8, but not IL-6 and TNF- $\alpha$, concentrations in bronchial secretions after just 4 days of treatment, and a significant reduction in 8-isoprostane (a product of lipid peroxidation) blood levels after 10 days when compared with matching placebo [31].

Since increased oxidative stress as expected in current smokers with mild-to-moderate COPD can affect $\beta_{2}$ adrenoceptor function, in a small study 30 current smokers suffering from non-reversible mild-to-moderate COPD received erdosteine $300 \mathrm{mg}$, NAC $600 \mathrm{mg}$ or placebo twice daily for 10 days [32]. Both erdosteine and NAC induced rapid and significant drops in ROS blood levels, but only erdosteine lowered 8-isoprostane levels and significantly restored short-term reversibility in COPD patients previously unresponsive to salbutamol. The kinetics of FEV changes was related to the corresponding changes in 8-isoprostane levels.

Another small study that enrolled 12 patients with moderate COPD who received erdosteine $300 \mathrm{mg}$ or placebo twice daily for 10 days evaluated the effects of erdosteine on eicosanoids [33]. Erdosteine caused a significant reduction in the levels of serum and urinary levels of LTB4. Furthermore, erdosteine also decreased blood ROS levels and slightly increased $\mathrm{FEV}_{1}$.

However, a trial that evaluated erdosteine given for 2 weeks in an open-label non-comparative study in 41 stable COPD patients with $\mathrm{FEV}_{1} / \mathrm{FVC}$ of $60 \pm 15 \%$ and $\geq 30 \mathrm{~mL}$ sputum per day showed that treatment with erdosteine was able to induce a significant clinical improvement without changes in spirometric tests and blood gases. Bronchodilators, used by $68 \%$ of patients, did not influence the results [34].

In a study in which patients with mild-to-moderate COPD were randomised to receive either placebo or two dosages of 


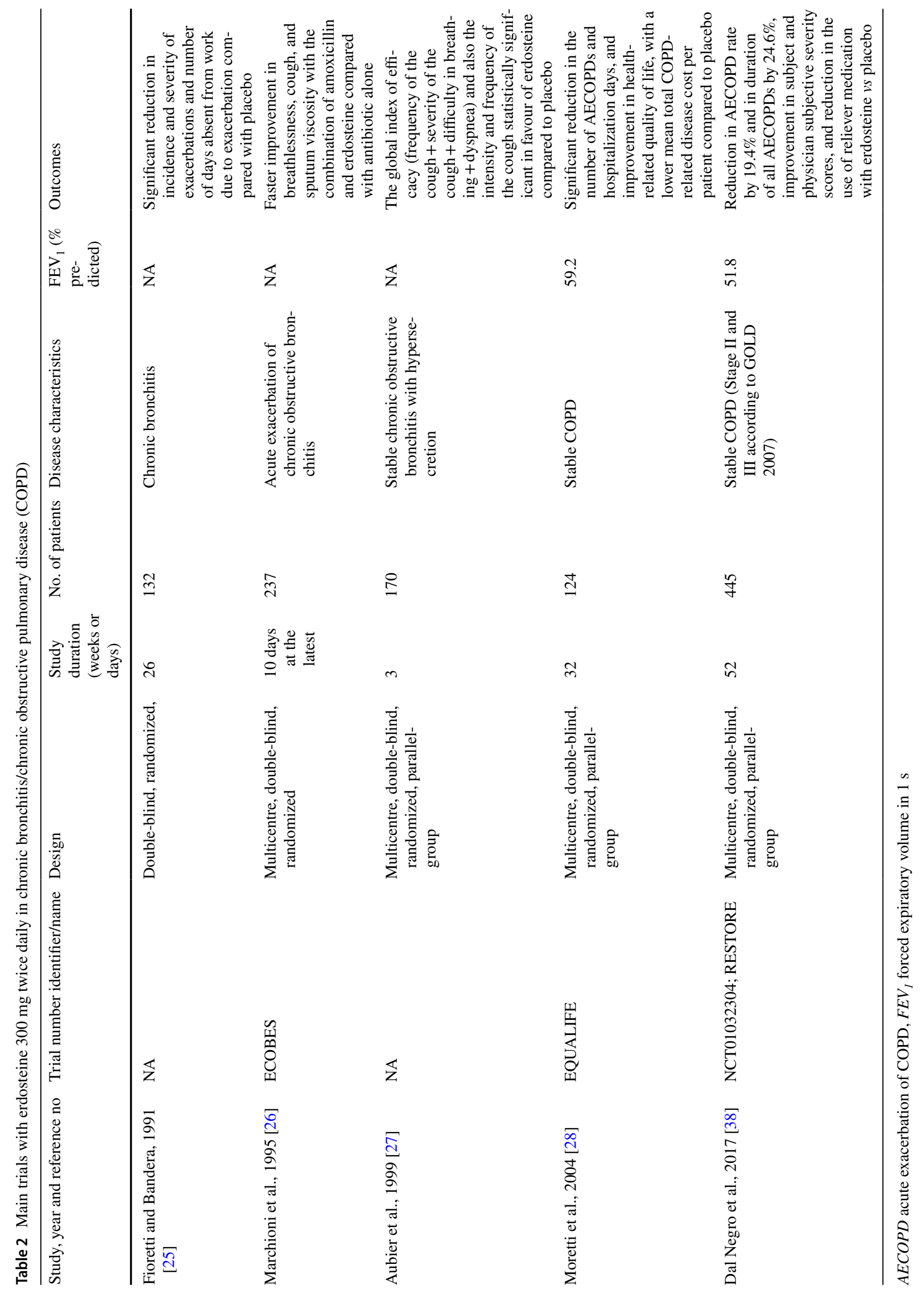


oral erdosteine (300 mg three times daily or $300 \mathrm{mg}$ twice daily) for 28 days in addition to their standard treatment, a dose-dependent decrease in ROS levels was observed from the second week [35]. This decrease became statistically different between the two doses of erdosteine in the fourth week of treatment and continued in the follow-up week. Erdosteine $900 \mathrm{mg} /$ day was also more effective than $600 \mathrm{mg} /$ day in lowering 8-isoprostane plasma levels and caused a greater improvement in $\% \mathrm{FEV}_{1}$ with salbutamol $400 \mu \mathrm{g}$ after 4 weeks of treatment.

The effect of erdosteine $(600 \mathrm{mg} /$ day $)$ or placebo administered for 10 days was also assessed on exercise-induced oxidative stress in 24 severe COPD patients [36]. Erdosteine improved the oxidant-antioxidant mismatch following physical exercise in these patients. After 10 days of treatment, ROS and 8-isoprostane plasma levels measured at rest (pre-exercise) were comparable in the two groups, but after a 6-min walk test the increases in ROS and 8-isoprostane plasma levels were lower in the group treated with erdosteine.

Two small groups of patients hospitalized for AECOPD were treated with standard therapy with corticosteroids, bronchodilators and antibiotics as appropriate plus erdosteine $900 \mathrm{mg}$ or a matching control, respectively, for 10 days [37]. In the group also treated with erdosteine there was greater reduction of the levels of serum C-reactive protein and improvements in symptom score and lung function. Furthermore, erdosteine was associated with a 39\% lower risk of exacerbations and a significant delay in time to first exacerbation compared with controls.

In 2017, the RESTORE (Reducing Exacerbations and Symptoms by Treatment with ORal Erdosteine in COPD) study was published [38]. This was a landmark study investigating the effects of a thiol-based drug in a population of 467 patients with COPD who were frequent exacerbators. To participate in this study, patients had to have experienced two or more AECOPDs requiring medical intervention in the previous 12 months, but no AECOPDs in the previous 2 months. After a 2-week run-in period, 228 patients received oral erdosteine at the approved dosage of $300 \mathrm{mg}$ twice daily and 239 were treated with placebo for 12 months in addition to their usual maintenance therapy for COPD. Erdosteine reduced the acute exacerbation rate by $19.4 \%$ (0.91 vs. 1.13 exacerbations/patient/year for erdosteine and placebo, respectively), mainly due to an effect on the rate of mild exacerbations ( 0.23 vs. 0.54 exacerbations/patient/ year for erdosteine and placebo, respectively). Interestingly, the acute exacerbation rate was 0.93 versus 1.16 in those using ICSs $(-19.5 \%)$ and 0.89 versus $1.10(-19.3 \%)$ in those who were not using ICSs. No significant difference was observed in the rate of moderate and severe exacerbations. However, erdosteine caused a $24.6 \%$ decrease in the duration of all exacerbations $(9.5 \pm 7.2$ days with erdosteine vs. 12.6 \pm 9.7 days with placebo) irrespective of event severity. Erdosteine was also able to significantly improve subject and physician subjective severity scores, and reduce the use of reliever medication.

A post hoc analysis of the 254 RESTORE patients with spirometrically defined moderate COPD (post-bronchodilator $\mathrm{FEV}_{1} 50-79 \%$ predicted), of which 126 received erdosteine $300 \mathrm{mg}$ twice daily and 128 placebo, reported not only a $47 \%$ reduction in the mean exacerbation rate with erdosteine compared to placebo $(0.27$ vs. 0.51 exacerbations per patient per year, respectively), and a $58.3 \%$ reduction in the mild exacerbation rate ( 0.23 vs. 0.53 mild exacerbations per patient per year), but also a shortened mean duration of exacerbations in erdosteine-treated patients (9.1 vs. 12.3 days for placebo), with significant reductions in the duration of mild and moderate-to-severe exacerbations [39]. Furthermore, the treatment with erdosteine was related to a prolonged mean time to first exacerbation (182 days for erdosteine vs. 169 days for placebo) and mean exacerbationfree time ( 279 days for erdosteine vs. 228 days for placebo). Interestingly, there was no differential effect of treatment based on eosinophil numbers. Furthermore, there was no difference in exacerbation frequency and duration between moderate COPD patients who were ICS users and non-users (Tables 3,4) and the effect of erdosteine on the time to first exacerbation was not influenced by the concomitant use of ICS (Table 5).

\section{Meta-Analyses Performed to Clarify the Role of Erdosteine in the Treatment of Patients with Chronic Bronchitis/ Chronic Obstructive Pulmonary Disease (COPD)}

In 2010, we performed a systematic review with meta-analysis to test the evidence available at the time for the efficacy of erdosteine in adults with stable or exacerbated chronic bronchitis/COPD [40]. We retrieved 31 potentially relevant studies, but had to exclude 16 of them from the analysis. The reasons for these exclusions were: assessment of mucus rheology only; dose-range-finding study; inadequate design; lack of symptom assessment up to 10 days; a different formulation of erdosteine; or insufficient efficacy information. Only one out of 15 selected randomized controlled trials (RCTs), which enrolled 1,046 adult patients, was published after 1996. Treatment with erdosteine was associated with a significant benefit in terms of symptom improvement, both versus placebo and versus other mucolytics. The findings of this study indicated that erdosteine plus antibiotics is more effective than antibiotic monotherapy in patients with chronic bronchitis/COPD, especially for the treatment of acute infective exacerbations, and supported the addition of 
erdosteine to the usual treatment in order to provide further resolution of symptoms and allow a more rapid recovery. However, the study also suggested that the benefit provided by erdosteine on cough and sputum scores was less evident in patients with stable chronic bronchitis/COPD, presumably due to the short duration of treatment in the analysed RCTs.

A further systematic review with meta-analysis of relevant studies published up to 31 July 2017 examined data on erdosteine from ten studies involving 1,278 patients (52.66\% in the active treatment group, $47.34 \%$ in the control group) with chronic bronchitis and/or COPD [41]. Treatment with erdosteine significantly improved the clinical score of patients affected by COPD and/or chronic bronchitis and reduced the overall risk of chronic bronchitis/COPD exacerbations and the risk of experiencing at least one exacerbation vs. control. In COPD patients receiving erdosteine, the time to the first exacerbation was significantly longer than in untreated subjects. Erdosteine was also better than control in influencing the duration of AECOPD and reducing the risk of hospitalization from COPD.

The Global Initiative for Chronic Obstructive Lung Disease (GOLD) 2020 states that mucolytic/antioxidant agents such as erdosteine, $S$-carboxymethylcysteine and NAC are to be used for patients who are not receiving ICSs because they may reduce the risk of acute exacerbation of COPD and improve health status [42]. However, no distinction is made between these three thiol-based drugs. We therefore performed pairwise and network meta-analyses to assess the efficacy of erdosteine, $S$-carboxymethylcysteine and NAC on AECOPD, duration of AECOPD and hospitalization [43]. We analysed data obtained from seven RCTs including 2753 COPD patients $(11.15 \%$ treated with erdosteine, $18.27 \%$ treated with $S$-carboxymethylcysteine, $20.41 \%$ treated with NAC and $50.16 \%$ treated with placebo). All three drugs significantly reduced the risk of AECOPD, but the network meta-analysis indicated that erdosteine was the most effective agent in preventing the risk of AECOPD, followed by $S$-carboxymethylcysteine and NAC. The personbased number needed-to-treat (NNT) analysis reported that 10.11 patients had to be treated with erdosteine for 1 year

Table 3 Effect of erdosteine on exacerbation rate in moderate chronic obstructive pulmonary disease (COPD) patients who were inhaled corticosteroid (ICS) users or non-users (modified from Calverley et al. [39])

\begin{tabular}{llllll}
\hline & \multicolumn{2}{l}{$\begin{array}{l}\text { Exacerbation rate/ } \\
\text { patient/year }\end{array}$} & & Effect size & \\
\cline { 2 - 3 } \cline { 5 - 6 } & Erdosteine & Placebo & & $\Delta$ vs placebo (\%) & $P$ value \\
\hline All patients & 0.27 & 0.51 & & -4.72 & 0.003 \\
Patients using ICS & 0.30 & 0.54 & & -44.2 & 0.002 \\
No ICS use & 0.26 & 0.49 & & -46.9 & 0.003 \\
\hline
\end{tabular}

Table 4 Effect of erdosteine on exacerbation duration in moderate chronic obstructive pulmonary disease (COPD) patients who were inhaled corticosteroid (ICS) users or non-users (modified from Calverley et al. [39])

\begin{tabular}{llllll}
\hline & \multicolumn{2}{l}{$\begin{array}{l}\text { Duration of exacer- } \\
\text { bation (days) }\end{array}$} & & Effect size & \\
\cline { 2 - 3 } \cline { 5 - 6 } \cline { 5 - 6 } & Erdosteine & Placebo & & $\Delta$ vs placebo (\%) & $P$ value \\
\hline All patients & 9.1 & 12.3 & & -26.0 & 0.02 \\
Patients using ICS & 9.3 & 12.5 & & -22.4 & 0.04 \\
No ICS use & 9.0 & 12.1 & & -25.6 & 0.02 \\
\hline
\end{tabular}

to prevent one AECOPD, compared to placebo, while the NNT values for both $S$-carboxymethylcysteine (30.92) and NAC (15.69) were not significantly different than placebo. Only erdosteine reduced the risk of experiencing at least one AECOPD and the risk of hospitalization due to AECOPD, while erdosteine and NAC both significantly reduced the duration of AECOPD. All three drugs were generally well tolerated.

\section{Further Potential Indications for Erdosteine in Pulmonary Medicine}

Although no clinical study has evaluated the addition of erdosteine to standard-of-care asthma medications, data from an experimental research in a guinea-pig model of allergic inflammation showed that 10 days treatment with erdosteine resulted in bronchodilation and stimulation of ciliary beat frequency, but did not affect the irritantinduced cough reflex, which is a positive finding because it indicates that erdosteine does not inhibit the expulsion of mucus from the respiratory tract by coughing [44]. Erdosteine demonstrated a modest decline in inflammatory cytokines IL-5, and IL-13 and an increase in the concentration of IL-10, cytokines known to be potent regulators of allergic inflammatory responses. These findings suggest the

Table 5 Effect of erdosteine on time to first exacerbation in moderate chronic obstructive pulmonary disease (COPD) patients who were inhaled corticosteroid (ICS) users or non-users (modified from Calverley et al. [39])

\begin{tabular}{llllll}
\hline & \multicolumn{2}{l}{$\begin{array}{l}\text { Time to first exacer- } \\
\text { bation (days) }\end{array}$} & & \% change & \\
\cline { 2 - 3 } & Erdosteine & Placebo & & $\Delta$ vs placebo (\%) & $P$ value \\
\hline All patients & $182 \pm 19$ & $169 \pm 25$ & +7.7 & $<0.001$ \\
Patients using & $180 \pm 23$ & $168 \pm 24$ & +7.1 & $<0.002$ \\
$\quad$ ICS & & & & $<0.003$ \\
No ICS use & $185 \pm 18$ & $170 \pm 23$ & +8.8 & $<$ \\
\hline
\end{tabular}


possibility that erdosteine may also be of benefit in patients with asthma as add-on therapy. In light of the documentation in patients with COPD that erdosteine shows benefit independently of corticosteroids, it would seem sensible to investigate the ability of this drug to act as a corticosteroidsparing agent.

Thiol-based drugs may also be useful for treating IPF because of the involvement of oxygen radicals and reduced GSH levels in IPF pathogenesis and progression [2]. Several experimental studies have shown that erdosteine is effective in preventing bleomycin-induced lung fibrosis in rats possibly via the repression of neutrophil accumulation, inhibition of lipid peroxidation, and maintenance of antioxidant and free radical scavenger properties [11, 45, 46]. There is also evidence that erdosteine particularly when combined with $S$-methyl-isothiourea sulfate, a nitric oxide synthase inhibitor, inhibits pulmonary inflammation, decreases IL-6, TNF- $\alpha$ and NF-kB production, and attenuates bleomycininduced pulmonary fibrosis [47]. This experimental evidence suggests that erdosteine merits further investigation in patients with pulmonary fibrosis to treat this life-threatening disease with a large unmet medical need.

\section{Conclusion}

There is solid evidence in the literature that erdosteine reduces the risk of AECOPD, although it is not yet possible to determine how much of this effect is related to its mucolytic action rather than to antioxidant, anti-inflammatory or antimicrobial activities, or to a combination of all of these. In effect, the documented multifaceted actions of erdosteine beyond mucolytic activity may be of relevance considering that AECOPDs differ in aetiology, being essentially infectious (viral, or bacterial, or both) or uninfectious, and also in biological substrate [48].

Bafadhel et al. identified four biologic cluster populations for exacerbation events [49]. Three clusters were termed as being 'bacteria-predominant', 'eosinophil-predominant' and 'virus-predominant'. A fourth cluster demonstrated low sputum mediator concentrations and had fewer events associated with known aetiology and was termed 'pauciinflammatory'. In each COPD patient who exhibits frequent exacerbations, a prevalent AECOPD biological substrate seems involved, which should be recognized and prevented accordingly [50]. Those with eosinophilic inflammation may benefit most from ICSs [51].

Erdosteine possesses several properties, such as potent antioxidant, anti-inflammatory and antibacterial activity against a variety of medically relevant bacteria, and is also able to influence bronchial tone. This makes erdosteine a valid preventive treatment of AECOPDs in the real world, where the biological substrate to be counteracted is almost never identified. There are no data on the ability of erdosteine to prevent viral episodes, but it is well known that thiol-based drugs are an effective strategy against influenza virus infection because virus-induced oxidative stress is important in the regulation of the host immune system [52].

It is particularly interesting that the RESTORE study found that the preventive effect on the risk of AECOPD exerted by erdosteine was present both in patients who were being treated with ICSs and in those who did no use this treatment, and, in any case, it was not affected by the blood count of eosinophils [38]. These findings clearly contrast with the GOLD strategy's approach to use erdosteine only in those COPD patients not treated simultaneously with an ICS [42]. Furthermore, they support the possibility of using erdosteine in a step-down approach, at least in patients with spirometrically defined moderate COPD [39]. This approach in COPD is characterized by the withdrawal of the ICS [53]. Withdrawal of ICS in patients under regular erdosteine did not significantly increase the rate of exacerbations [38, 39], although clinically a risk of exacerbation is still present when ICSs are discontinued [54]. It is likely that the addition of erdosteine could minimize this risk, but further evidence generated by an expressly designed study to confirm this possibility is now required.

\section{Declarations}

Conflict of interest Mario Cazzola, Paola Rogliani, Luigino Calzetta and Clive Page are consultants to Recipharm, which manufactures erdosteine.

Funding Open access funding provided by Università degli Studi di Roma Tor Vergata within the CRUI-CARE Agreement. This article was not funded/sponsored, and no writing assistance was utilized in its production.

Ethics approval Not applicable.

Consent to participate Not applicable.

Consent for publication Not applicable.

Code availability Not applicable.

Open Access This article is licensed under a Creative Commons Attribution-NonCommercial 4.0 International License, which permits any non-commercial use, sharing, adaptation, distribution and reproduction in any medium or format, as long as you give appropriate credit to the original author(s) and the source, provide a link to the Creative Commons licence, and indicate if changes were made. The images or other third party material in this article are included in the article's Creative Commons licence, unless indicated otherwise in a credit line to the material. If material is not included in the article's Creative Commons licence and your intended use is not permitted by statutory regulation or exceeds the permitted use, you will need to obtain permission directly from the copyright holder. To view a copy of this licence, visit http://creativecommons.org/licenses/by-nc/4.0/. 


\section{References}

1. Dechant KL, Noble S. Erdosteine. Drugs. 1996;52(6):875-82.

2. Cazzola M, Calzetta L, Page C, Rogliani P, Matera MG. Thiolbased drugs in pulmonary medicine: much more than mucolytics. Trends Pharmacol Sci. 2019;40(7):452-63.

3. Miyake K, Kaise T, Hosoe H, Akuta K, Manabe H, Ohmori K. The effect of erdosteine and its active metabolite on reactive oxygen species production by inflammatory cells. Inflamm Res. 1999;48(4):205-9.

4. Hosoe H, Kaise T, Ohmori K, et al. Mucolytic and antitussive effects of erdosteine. J Pharm Pharmacol. 1999;51(8):959-66.

5. Hattori M. Mucociliary function of chronic inflammation in upper and lower airways. Auris Nasus Larynx. 1994;21(4):219-25.

6. Hosoe H, Kaise T, Ohmori K. Erdosteine enhances mucociliary clearance in rats with and without airway inflammation. J Pharmacol Toxicol Methods. 1998;40(3):165-71.

7. Hosoe H, Kaise T, Ohmori K. Effects on the reactive oxygen species of erdosteine and its metabolite in vitro. Arzneimittelforschung. 2002;52(6):435-40.

8. Braga PC, Dal Sasso M, Zuccotti T. Assessment of the antioxidant activity of the SH metabolite I of erdosteine on human neutrophil oxidative bursts. Arzneimittelforschung. 2000;50(8):739-46.

9. Marabini L, Calò R, Braga PC. Protective effect of erdosteine metabolite I against hydrogen peroxide-induced oxidative DNA-damage in lung epithelial cells. Arzneimittelforschung. 2011;61(12):700-6.

10. Braga PC, Culici M, Dal Sasso M, Falchi M, Spallino A. Free radical scavenging activity of erdosteine metabolite I investigated by electron paramagnetic resonance spectroscopy. Pharmacology. 2010;85(4):195-202.

11. Boyaci H, Maral H, Turan G, et al. Effects of erdosteine on bleomycin-induced lung fibrosis in rats. Mol Cell Biochem. 2006;281(1-2):129-37.

12. Demiralay R, Gürsan N, Ozbilim G, Erdogan G, Demirci E. Comparison of the effects of erdosteine and $\mathrm{N}$-acetylcysteine on apoptosis regulation in endotoxin-induced acute lung injury. J Appl Toxicol. 2006;26(4):301-8.

13. Erdem A, Gedikli E, Yersal N, et al. Protective role of erdosteine pretreatment on oleic acid-induced acute lung injury. J Surg Res. 2017;213:234-42.

14. Lee IT, Yang CM. Role of NADPH oxidase/ROS in pro-inflammatory mediators-induced airway and pulmonary diseases. Biochem Pharmacol. 2012;84(5):581-90.

15. Park JS, Park MY, Cho YJ, et al. Anti-inflammatory effect of erdosteine in lipopolysaccharide-stimulated RAW 264.7 cells. Inflammation. 2016;39(4):1573-81.

16. Jang YY, Song JH, Shin YK, Han ES, Lee CS. Depressant effects of ambroxol and erdosteine on cytokine synthesis, granule enzyme release, and free radical production in rat alveolar macrophages activated by lipopolysaccharide. Pharmacol Toxicol. 2003;92(4):173-9.

17. Hayashi K, Hosoe H, Kaise T, Ohmori K. Protective effect of erdosteine against hypochlorous acid-induced acute lung injury and lipopolysaccharide-induced neutrophilic lung inflammation in mice. J Pharm Pharmacol. 2000;52(11):1411-6.

18. Demiralay R, Gürsan N, Erdem H. Regulation of sepsis-induced apoptosis of pulmonary cells by posttreatment of erdosteine and $\mathrm{N}$-aceylcysteine. Toxicology. 2006;228(2-3):151-61.

19. Braga PC, Dal Sasso M, Culici M, Verducci P, Lo Verso R, Marabini L. Effect of metabolite I of erdosteine on the release of human neutrophil elastase. Pharmacology. 2006;77(3):150-4.

20. Braga PC, Dal Sasso M, Sala MT, Gianelle V. Effects of erdosteine and its metabolites on bacterial adhesiveness. Arzneimittelforschung. 1999;49(4):344-50.
21. Braga PC, Zuccotti T, Dal Sasso M. Bacterial adhesiveness: effects of the SH metabolite of erdosteine (mucoactive drug) plus clarithromycin versus clarithromycin alone. Chemotherapy. 2001;47(3):208-14.

22. Dal Sasso M, Bovio C, Culici M, Braga PC. The combination of the SH metabolite of erdosteine (a mucoactive drug) and ciprofloxacin increases the inhibition of bacterial adhesiveness achieved by ciprofloxacin alone. Drugs Exp Clin Res. 2002;28(2-3):75-82.

23. Irwin RS, Boulet LP, Cloutier MM, et al. Managing cough as a defense mechanism and as a symptom. A consensus panel report of the American College of Chest Physicians. Chest. 1998;114(2 Suppl Managing):133S-81S.

24. Bolser DC. Cough suppressant and pharmacologic protussive therapy: ACCP evidence-based clinical practice guidelines. Chest. 2006;129(1 Suppl):238S-49S.

25. Fioretti M, Bandera M. Prevention of exacerbations in chronic bronchitic patients with erdosteine. Med Prax. 1991;12(4):219-27.

26. Marchioni CF, Polu JM, Taytard A, Hanard T, Noseda G, Mancini C. Evaluation of efficacy and safety of erdosteine in patients affected by chronic bronchitis during an infective exacerbation phase and receiving amoxycillin as basic treatment (ECOBES, European Chronic Obstructive Bronchitis Erdosteine Study). Int J Clin Pharmacol Ther. 1995;33(11):612-8.

27. Aubier M, Berdah L. Etude multicentrique, contrôlée, en double aveugle, de l'efficacité et de la tolérance de Vectrine (erdostéine) versus placebo dans le traitement de la bronchite chronique hypersecrétante stabilisée. Rev Mal Respir. 1999;16(4):521-8.

28. Moretti M, Bottrighi P, Dallari R, et al. The effect of longterm treatment with erdosteine on chronic obstructive pulmonary disease: the EQUALIFE Study. Drugs Exp Clin Res. 2004;30(4):143-52.

29. Crisafulli E, Coletti O, Costi S, et al. Effectiveness of erdosteine in elderly patients with bronchiectasis and hypersecretion: a 15-day, prospective, parallel, open-label, pilot study. Clin Ther. 2007;29(9):2001-9.

30. Basyigit I, Yildiz F, Cekmen M, Duman C, Bulut O. Effects of erdosteine on smoking-induced lipid peroxidation in healthy smokers. Drugs R D. 2005;6(2):83-9.

31. Dal Negro RW, Visconti M, Micheletto C, Tognella S. Changes in blood ROS, e-NO, and some pro-inflammatory mediators in bronchial secretions following erdosteine or placebo: a controlled study in current smokers with mild COPD. Pulm Pharmacol Ther. 2008;21(2):304-8.

32. Dal Negro R, Visconti M, Trevisan F, Bertacco S, Micheletto C, Tognella S. Erdosteine enhances airway response to salbutamol in patients with mild-to-moderate COPD. Ther Adv Respir Dis. 2008;2(5):271-7.

33. Dal Negro RW, Visconti M, Tognella S, Micheletto C. Erdosteine affects eicosanoid production in COPD. Int J Clin Pharmacol Ther. 2011;49(1):41-5.

34. de Castro Pereira CA, Cardoso AP, Cavallazzi AC, Pinheiro VGF, de Oliveira MVC, Esposito C. Eficácia e tolerabilidade da erdosteína na doença pulmonar obstrutiva crônica. Rev Bras Med. 2000;57(5):481-5.

35. Dal Negro RW, Visconti M, Turco P. Efficacy of erdosteine 900 versus $600 \mathrm{mg} /$ day in reducing oxidative stress in patients with COPD exacerbations: results of a double blind, placebo-controlled trial. Pulm Pharmacol Ther. 2015;33:47-51.

36. Dal Negro RW, Visconti M. Erdosteine reduces the exerciseinduced oxidative stress in patients with severe COPD: results of a placebo-controlled trial. Pulm Pharmacol Ther. 2016;41:48-51.

37. Moretti M, Fagnani S. Erdosteine reduces inflammation and time to first exacerbation postdischarge in hospitalized patients with AECOPD. Int J Chron Obstruct Pulmon Dis. 2015;10:2319-25. 
38. Dal Negro RW, Wedzicha JA, Iversen M, et al. Effect of erdosteine on the rate and duration of COPD exacerbations: the RESTORE study. Eur Respir J. 2017;50(4):1700711.

39. Calverley PM, Page C, Dal Negro RW, et al. Effect of erdosteine on COPD exacerbations in COPD patients with moderate airflow limitation. Int J Chron Obstruct Pulmon Dis. 2019;14:2733-44.

40. Cazzola M, Floriani I, Page CP. The therapeutic efficacy of erdosteine in the treatment of chronic obstructive bronchitis: a meta-analysis of individual patient data. Pulm Pharmacol Ther. 2010;23(2):135-44.

41. Cazzola M, Calzetta L, Page C, Rogliani P, Matera MG. Impact of erdosteine on chronic bronchitis and COPD: a meta-analysis. Pulm Pharmacol Ther. 2018;48:185-94.

42. GOLD. Global strategy for the diagnosis, management and prevention of COPD, Global Initiative for Chronic Obstructive Lung Disease (GOLD). 2020 report. https://www.goldcoped.org/. Accessed 28 May 2020.

43. Rogliani P, Matera MG, Page C, Puxeddu E, Cazzola M, Calzetta L. Efficacy and safety profile of mucolytic/antioxidant agents in chronic obstructive pulmonary disease: a comparative analysis across erdosteine, carbocysteine, and $\mathrm{N}$-acetylcysteine. Respir Res. 2019;20(1):104.

44. Fraňová S, Kazimierová I, Pappová L, Molitorisová M, Jošková M, Šutovská M. The effect of erdosteine on airway defence mechanisms and inflammatory cytokines in the settings of allergic inflammation. Pulm Pharmacol Ther. 2019;54:60-7.

45. Sogut S, Ozyurt H, Armutcu F, et al. Erdosteine prevents bleomycin-induced pulmonary fibrosis in rats. Eur $\mathrm{J}$ Pharmacol. 2004;494(2-3):213-20
46. Yildirim Z, Kotuk M, Iraz M, et al. Attenuation of bleomycininduced lung fibrosis by oral sulfhydryl containing antioxidants in rats: erdosteine and $\mathrm{N}$-acetylcysteine. Pulm Pharmacol Ther. 2005;18(5):367-73.

47. Guzel A, Kayhan S, Tutuncu S, et al. Attenuation of bleomycin induced lung fibrosis by erdosteine and inhibition of the inducible nitric oxide synthase. Bratisl Lek Listy. 2015;116(3):196-202.

48. Cazzola M, Calzetta L, Rogliani P, Matera MG. The challenges of precision medicine in COPD. Mol Diagn Ther. 2017;21(4):345-55.

49. Bafadhel M, McKenna S, Terry S, et al. Acute exacerbations of chronic obstructive pulmonary disease: identification of biologic clusters and their biomarkers. Am J Respir Crit Care Med. 2011;184(6):662-71.

50. Tantucci C, Pini L. Inhaled corticosteroids in COPD: trying to make a long story short. Int J Chron Obstruct Pulmon Dis. 2020;15:821-9.

51. Pavord ID, Lettis S, Locantore N, et al. Blood eosinophils and inhaled corticosteroid/long-acting $\beta$-2 agonist efficacy in COPD. Thorax. 2016;71(2):118-25.

52. Liu M, Chen F, Liu T, Chen F, Liu S, Yang J. The role of oxidative stress in influenza virus infection. Microbes Infect. 2017;19(12):580-6.

53. Cazzola M, Rogliani P, Matera MG. Escalation and de-escalation of therapy in COPD: myths, realities and perspectives. Drugs. 2015;75(14):1575-85.

54. Calzetta L, Matera MG, Braido F, et al. Withdrawal of inhaled corticosteroids in COPD: a meta-analysis. Pulm Pharmacol Ther. 2017;45:148-58. 